\title{
Carbon nanotubes - chitosan nanobiocomposite for immunosensor
}

\author{
Ajeet Kaushik ${ }^{\text {a,b }}$, Pratima R. Solanki ${ }^{a}$, M.K. Pandey ${ }^{a}$, Keiichi Kaneto ${ }^{c}$, Sharif Ahmad ${ }^{\text {b }}$, Bansi D. Malhotra ${ }^{\text {a, }}$ \\ a Department of Science and Technology Centre on Biomolecular Electronics, National Physical Laboratory, New Delhi-110012, India \\ b Materials Research Laboratory, Department of Chemistry, Jamia Millia Islamia, New Delhi-110025, India \\ ' Graduate School of Life Science and Systems Engineering, Kyushu Institute of Technology, Kitakyushu, 808-0196, Japan
}

\begin{abstract}
A B S T R A C T
Carboxylic group functionalized single walled (SW) and multi walled (MW) carbon nanotubes (CNT) have been incorporated into biopolymer matrix of chitosan $(\mathrm{CH})$ to fabricate nanobiocomposite film onto indiumtin-oxide (ITO) coated glass plate for co-immobilization of rabbit-immunoglobulin ( $\mathrm{r}$-IgGs) and bovine serum albumin (BSA) to detect ochratoxin-A (OTA). The results of electrochemical studies reveal that presence of both CNT results in increased electro-active surface area of $\mathrm{CH}$ leading to enhanced electron transport in these nanobiocomposites. Moreover, in CH-SWCNT and CH-MWCNT nanobiocomposites the availability of $\mathrm{NH}_{2} / \mathrm{OH}$ group in $\mathrm{CH}$ and surface charged CNT also increases loading of the r-IgGs resulting in enhanced electron transport responsible for improved sensing characteristics. Compared to BSA/r-IgGs/CHMWCNT/ITO immunoelectrode, electrochemical response studies of BSA/r-IgGs/CH-SWCNT/ITO immunoelectrode carried out as a function of OTA concentration exhibits improved linearity as $0.25-6 \mathrm{ng} / \mathrm{dL}$, detection limit as $0.25 \mathrm{ng} / \mathrm{dL}$, response time as $25 \mathrm{~s}$, and sensitivity as $21 \mu \mathrm{Ang} \mathrm{dL}{ }^{-1} \mathrm{~cm}^{-2}$ with the regression coefficient as 0.998 .
\end{abstract}

\section{Introduction}

Carbon nanotubes (CNT) due to their unique internal structure, electrical conductivity, high chemical activity, low mass density, high electro-active surface area, thermal stability and high mechanical strength have recently aroused much interest for the development of novel technological applications such as batteries, nano-electronic devices, fuel cells, as probe tips in scanning probe microscopy, supercapacitors, nano-transistors, electrochemical actuators, gas/bio sensors etc, [1,2]. Recently, surface-confined CNT due to their ability to promote electron transfer reactions with electro-active species have attracted much interest as potential candidates for the development of nanoscale electrochemical biosensors [3-12]. The structure-dependent metallic character of CNT should allow promotion of electron transfer reactions at low over potential. This characteristic along with the high surface area provides the favourable environment to immobilize desired biomolecules for unique biochemical sensing systems. The recent advances in increased production of well-controlled aligned carbon nanotube arrays have led to their application in biosensors technology [10].

CNT have been used as components in biological devices. However, the current focus is on attaching desired biomolecules onto CNT for biomedical applications.
It has been shown that small proteins can be entrapped into the inner channel of opened by adsorption via electrostatic interactions. The attachment of small proteins on the outer surface of CNT has been achieved, either by hydrophobic and electrostatic interactions via covalent bonding or by functionalization of the nanotube sides. CNT have the ability to accelerate electron transfer reactions involving different biomolecules such as dopamine [14], $\beta$-nicotinamide adenine dinucleotide (NADH) [15], norepinephrine [16], cytochrome c [17], ascorbic acid [18], 5-hydroxytrptamine [19], cysteine [20], homocysteine [21], nucleic acids [22] and cholesterol [12].

The aggregation of CNT due to their high surface free energy and existence of inter-tube vander Waals forces has limited their technological potential. The poor solubility of CNT in most solvents is a major barrier to development of CNT based nanodevices. Therefore, intrinsically hydrophobic surface of CNT can be functionalized covalently or non-covalently for obtaining increased biocompatibility, solubility and drug-loading capacity $[12,13]$ for bio- medical applications.

These problems can perhaps be overcome by covalent attachment of CNT with alkyl chains and by non-covalent functionalization of CNT with conducting/bio polymers by preparing nanocomposites via hydrophobic or $\pi-\pi$ interactions. The first approach disrupts the extended $\pi$-network at CNT surfaces resulting in their poor mechanical and electrical properties and the incorporation of CNT with polymeric backbone can be useful to improve solubility of CNT without changing their physical properties $[12,13]$.

Conducting/bio polymer-CNT nanocomposites have been found to exhibit the properties of both the parent precursors along with its synergetic effect that is helpful for biosensor application [12,13,23- 
25]. To optimize the use of CNT for bio-technological applications, it is necessary to functionalize CNT with biomaterials including biomolecules, biopolymers and other bio-nanostructures. Among the various biomaterials, chitosan $(\mathrm{CH})$ a biopolymer is derived by the alkaline deacetylation of chitin found in the exoskeletons of shrimps and crabs. $\mathrm{CH}$ due to availability of amine/hydroxyl groups, non-toxicity, biocompatibility and excellent film can be used for wound dressings, carriers for drug delivery systems, artificial skin, space filling implants and most importantly to immobilize desired biomolecules for biosensor application [27]. In spite of these developments, there is a considerable scope to improve optical and electrical properties of $\mathrm{CH}$ via incorporation of $\mathrm{CNT}$ in the $\mathrm{CH}$ matrix to prepare nanobiocomposites for biosensing applications.

Zhang et al. [28] have fabricated CNT-CH platform for electrochemical sensing based on dehydrogenase enzymes. Luo et al. [25] have electrochemically deposited $\mathrm{CH}-\mathrm{MWCNT}$ nanocomposite to obtain excellent electro catalytic ability in the reduction and oxidation of hydrogen peroxide $\left(\mathrm{H}_{2} \mathrm{O}_{2}\right)$. Zhang et al. [26] have prepared electrochemical sensing platform based on the $\mathrm{CNT} /$ toluidine blue $\mathrm{O}-\mathrm{CH} / \mathrm{CH}$ system to facilitate electron transfer processes. DNA biosensors based on $\mathrm{CH}$ film doped with CNT has been prepared by Li et al. [29]. Liu et al. [30] have also studied direct electron transfer of glucose oxidase (GOx) in the CNT-CH nanocomposite matrix. Cui et al. [31] have prepared a novel $\mathrm{CH} /$ polyvinylimidazole-Os/CNT/lactate oxidase nanocomposite bioelectrode onto gold electrode for lactate detection. Wang et al. [32] have studied the fabrication of copper nanoparticle-CH-CNT/GCE for electrochemical sensing of $\mathrm{H}_{2} \mathrm{O}_{2}$ and glucose. Solanki et al. [33] have prepared nanobiocomposite film of $\mathrm{MWCNT} / \mathrm{CH}-\mathrm{SiO}_{2}$ for the co-immobilization of cholesterol oxidase and cholesterol esterase to detect total cholesterol. Lin et al. [34] have prepared a sensitive amperometric immunosensor for $\alpha$-fetoprotein in human serum based on CNT/gold nanoparticle/CH film onto GCE. In spite of these developments $\mathrm{CH}-\mathrm{CNT}$ nanobiocomposite has not yet been explored for immunosensor application.

We report results of studies relating to the co-immobilization of r-IgGs and BSA onto $\mathrm{COOH}-\mathrm{CNTS}$ and $\mathrm{CH}$ based nanobiocomposite films for ochratoxin-A (OTA) detection. OTA is the most abundant food contaminating mycotoxin found in tissues and organs of animals and human and is known to produce nephrotoxic, teratogenic, carcinogenic (Group 2B) and immune toxic activity in living beings, through consumption of improperly stored food products [35,36].

\section{Experimental details}

\subsection{Materials}

Chitosan (Mw $2.4 \times 10^{-6}$ ), ochratoxin-A (Aspergillus ochraceus), rabbit immunoglubin antibodies ( $\mathrm{r}$-IgGs), and bovine albumin serum (BSA; 98\% purity) have been purchased from Sigma Aldrich. All these chemicals are of analytical grade and have been used without further purification. Indium-tin-oxide (ITO) coated glass plates have been obtained from Balzers, UK. The deionized water (resistance $\sim 18 \mathrm{M} \Omega$ ) from Millipore water purification system (Milli Q $10 \mathrm{TS}$ ) has been used for the preparation of the solutions and buffers.

\subsection{Preparation of $\mathrm{CH}-\mathrm{SWCNTS} / \mathrm{ITO}$ and $\mathrm{CH}-\mathrm{MWCNTS} / \mathrm{ITO}$ nanobiocomposite electrode}

Single walled carbon nanotubes (SWCNT) have been synthesized using the arc-discharge process using graphite/catalyst [nickel (8.6 wt.\%), cobalt (8.6wt.\%)] composite electrode [37]. Multiwall carbon nanotubes (MWCNT) (90\%) have been synthesized by catalytic chemical vapour deposition using a mixture of ferrocene and toluene as a source of catalyst and hydrocarbon, respectively [38]. These SWCNTs and MWCNT are then purified and functionalized ( $\mathrm{COOH}$ group) and refluxed with concentrated boiling nitric acid for
$12 \mathrm{~h}$, and then washed with distilled water followed by rinsing with ethanol and drying at $60^{\circ} \mathrm{C}$ [39].

The calculated amount of SWCNT ( $1 \mathrm{mg}$ ) and MWCNTs ( $1 \mathrm{mg}$ ) are dispersed in $\mathrm{CH}(0.50 \%)$ solution [prepared by dissolving $\mathrm{CH}(50 \mathrm{mg})$ in $100 \mathrm{~mL}$ of acetate buffer $(0.05 \mathrm{M}, \mathrm{pH} 4.2)$ ] by sonication for $12 \mathrm{~h}$ at room temperature $\left(25^{\circ} \mathrm{C}\right)$. The film of $\mathrm{CH}-\mathrm{SWCNT}$ and $\mathrm{CH}-\mathrm{MWCNT}$ nanobiocomposites are fabricated by dispersing $10 \mu \mathrm{L}$ solution of both the nanobiocomposites onto an ITO $\left(0.25 \mathrm{~cm}^{2}\right)$ surface allowing it to dry at room temperature for about $12 \mathrm{~h}$ in a controlled environment. These CH-SWCNT/ITO and CH-MWCNT/ITO nanobiocomposite films are washed with deionized water to remove unbound particles.

The electrochemical experiments using both $\mathrm{CH}-\mathrm{SWCNT} / \mathrm{ITO}$ and CH-MWCNT/ITO nanobiocomposite electrodes have been repeated in multiple sets and the results are reproducible within $1 \%$ variation indicating that both the nanobiocomposites are stable and can be used more than 25 times.

\subsection{Immobilization of r-IgGs onto CH-SWCNT/ITO and CH-MWCNT/ITO nanobiocomposite electrode}

OTA (A. ochraceus) solution is prepared in phosphate buffer ( $50 \mathrm{mM}, \mathrm{pH} 7.0$ ) with $10 \%$ methanol. Solution of rabbit antibodies ( $\mathrm{r}-$ IgGs) is prepared in phosphate buffer ( $50 \mathrm{mM}, \mathrm{pH} 7.0$ ). Both the solutions containing $0.15 \mathrm{M} \mathrm{NaN}_{3}$ as a preservative are aliquoated and stored at $-20^{\circ} \mathrm{C}$. The immobilization of $\mathrm{r}-\operatorname{IgG}$ has been carried out by spreading $10 \mu \mathrm{L}$ solution onto the $\mathrm{CH}-\mathrm{CNT}$ electrode. Bovine serum albumin (BSA, $98 \%$ ) dissolved in phosphate buffer ( $50 \mathrm{mM}, \mathrm{pH} 7.0)$ is used as the blocking agent for non-specific binding sites.

\subsection{Characterization}

The FTIR spectra have been recorded on Perkin Elmer, Spectrum BX II spectrophotometer. Autolab Potentiostat/Galvanostat (Eco Chemie, Netherlands) is used for electrochemical studies such as cyclic voltammetry, differential pulse voltammetry and electrochemical impedance spectroscopy. These electrochemical measurements are carried out using a three-electrode cell with $\mathrm{CH}-\mathrm{SW} / \mathrm{MWCNT} / \mathrm{ITO}$ nanobiocomposite electrode and BSA/r-IgGs/CH-SW/MW CNT/ITO immunoelectrode as the working electrode, platinum wire as the counter electrode, and saturated $\mathrm{Ag} / \mathrm{AgCl}$ electrode as a reference electrode in phosphate buffer saline [PBS ( $50 \mathrm{mM}, \mathrm{pH} 7.0,0.9 \% \mathrm{NaCl})$ ] containing $5 \mathrm{mM}[\mathrm{Fe}(\mathrm{CN}) 6]^{3-/ 4-}$.

\section{Results and discussion}

\subsection{Fourier transform infrared spectroscopic studies}

The functionalization of CNT, formation of $\mathrm{CH}-\mathrm{CNT}$ nanobiocomposite and immobilization of r-IgGs and BSA onto $\mathrm{CH}-\mathrm{CNT}$ nanobiocomposite films have been studied using Fourier transforms infrared (FTIR) spectroscopy (Fig. 1). IR spectra of SWNT (curve a, Fig. 1A) and MWCNT (curve b, Fig. 1A) exhibit bands that correspond to the functional groups of $\mathrm{COOH}-\mathrm{CNT}$. Both of the curves show peak at $1620 \mathrm{~cm}^{-1}$ due to the $\mathrm{C}-\mathrm{C}$ graphitic stretching mode that is infrared-activated by extensive side-wall functionalization. The band at $1385 \mathrm{~cm}^{-1}$ is assigned to $\mathrm{O}-\mathrm{H}$ deformation vibration mode due to adsorbed water, $\mathrm{COOH}$ group and partial oxidation of CNT, while in curve (b) the absorbance band at $1385 \mathrm{~cm}^{-1}$ is attributed to O-H deformation vibration of absorption water and $-\mathrm{COOH}$, the band at $1100 \mathrm{~cm}^{-1}$ is assigned to the $\mathrm{C}-\mathrm{O}$ stretching vibration. The weak band at $1740 \mathrm{~cm}^{-1}$ is attributed to the carbonyl stretching vibration in the $-\mathrm{COOH}$ groups reveals the $\mathrm{COOH}$ functionalization of CNT [39]. However, the IR bands in the spectra of SWCNT are well-resolved and are sharper than that of the MWCNT due to the open frame network of SWCNT resulting in improved electrical properties. 

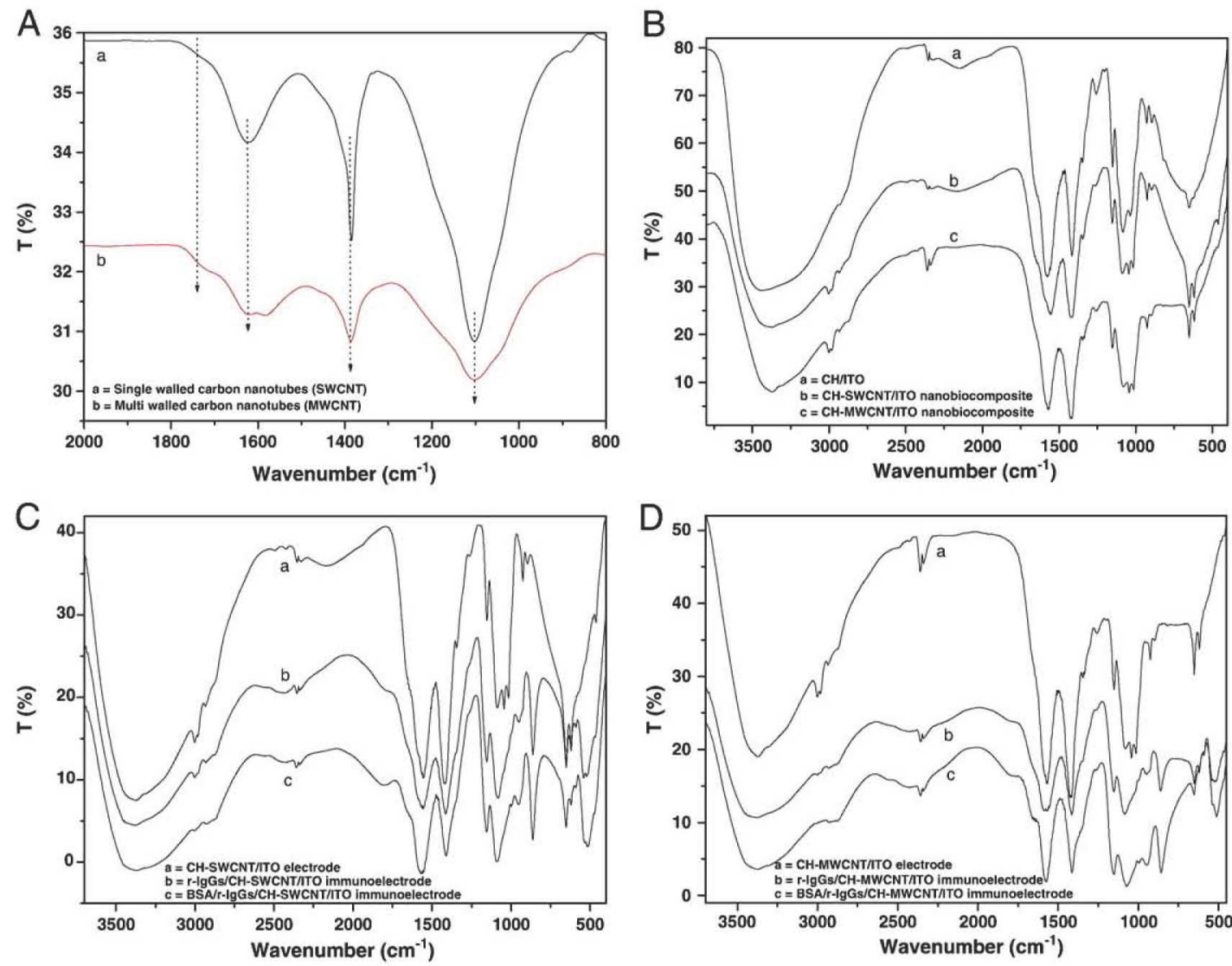

E

BSA/r-lgGs/CH-CNT/ITO immunosensor

\section{CH-CNT/ITO Nanobiocomposite}
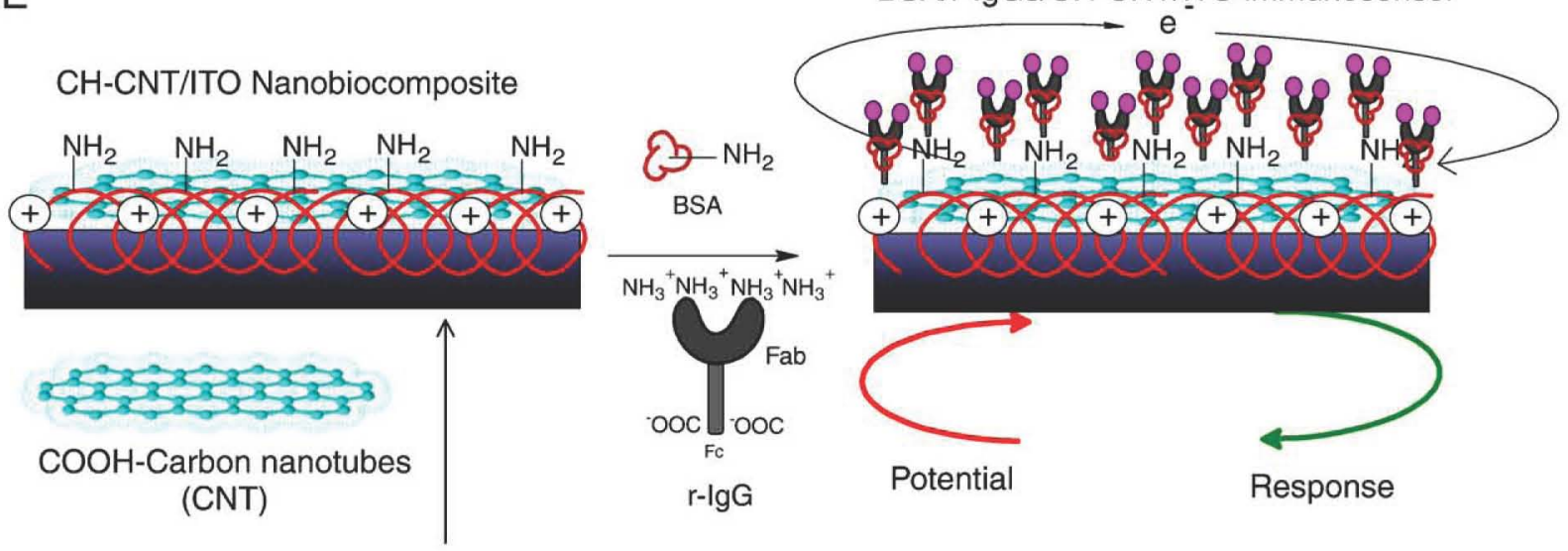

BSA

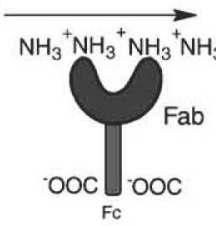

$r-\lg G$

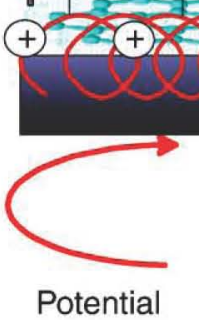

Potential

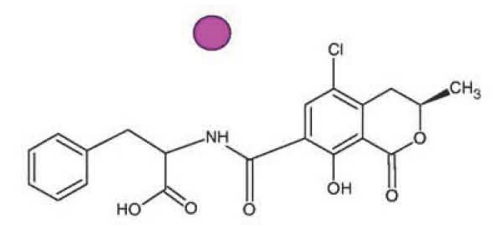

Ochratoxin-A (OTA)

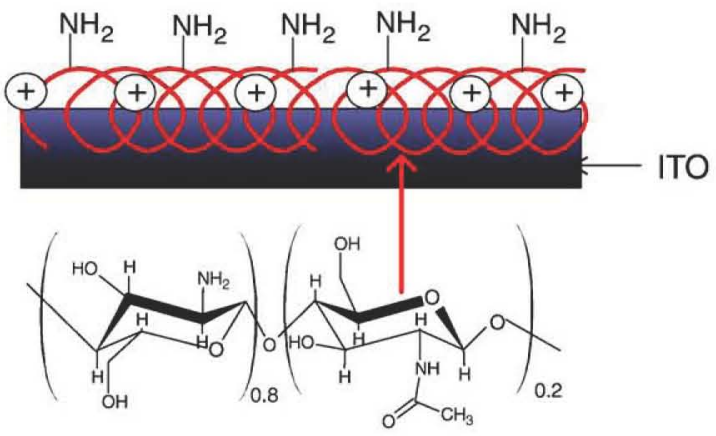

Chitosan $(\mathrm{CH})$

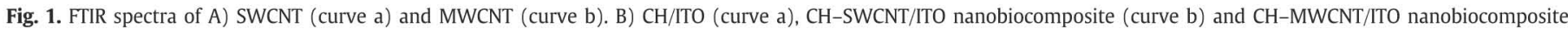

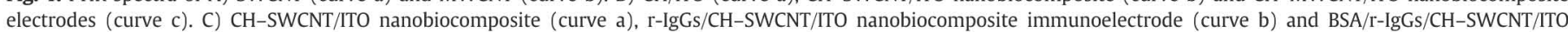

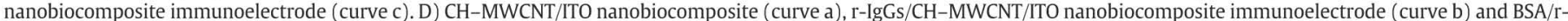

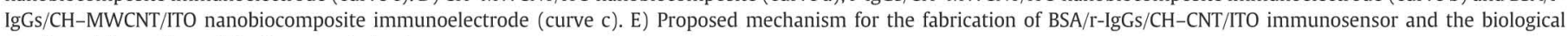
reaction at the surface of the immunoelectrode. 
FTIR spectra of $\mathrm{CH}$ (curve a, Fig. 1B) the IR band at bands at 3150 $3500 \mathrm{~cm}^{-1}$ is due to the stretching vibration mode of $\mathrm{OH}$ and $\mathrm{NH}_{2}$ group, $1650 \mathrm{~cm}^{-1}$ is due to the amide I group (C-O stretching along with $\mathrm{N}-\mathrm{H}$ deformation mode), $1570 \mathrm{~cm}^{-1}$ peak is attributed to the $\mathrm{NH}_{2}$ group due to $\mathrm{N}-\mathrm{H}$ deformation, $1425 \mathrm{~cm}^{-1}$ peak is due to $\mathrm{C}-\mathrm{N}$ axial deformation (amine group band), $1341 \mathrm{~cm}^{-1}$ peak is due to the $\mathrm{COO}^{-}$ group in carboxylic acid salt, $1150 \mathrm{~cm}^{-1}$ is assigned to the special broad peak of $\beta$ (1-4) glucosidic band in polysaccharide unit, $1086 \mathrm{~cm}^{-1}$ is attributed to the stretching vibration mode of the hydroxyl group, $1020 \mathrm{~cm}^{-1}$ is due to the stretching vibration of $\mathrm{C}-\mathrm{O}-\mathrm{C}$ in the glucose circle. The band at $1060-1015 \mathrm{~cm}^{-1}$ is correspond to $\mathrm{CH}-\mathrm{OH}$ in cyclic compounds. The IR corresponds to $\mathrm{NH}_{2} / \mathrm{OH}$ group [40].

Fig. 1B shows the FTIR spectra of $\mathrm{CH} / \mathrm{ITO}$ electrode (curve a), CH-SWCNT/ITO nanobiocomposite electrode (curve b) and CH-MWCNT/ ITO nanobiocomposite electrode (curve c). The IR band of CH and SWCNT/ MWCNT overlap in the spectrum of CH-SWCNT (curve b, Fig. 1B) and $\mathrm{CH}_{-}$ SWCNT (curve c, Fig. 1B) nanobiocomposites respectively, because the functional group corresponds to CNTs and $\mathrm{CH}$ shows the IR bands in the same region. However, the shape and peak position of the band corresponds to the $\mathrm{NH}_{2} / \mathrm{OH}$ groups of the $\mathrm{CH}$ changes in the spectra of $\mathrm{CH}-\mathrm{SWCNT}$ and $\mathrm{CH}-\mathrm{MWCNT}$ nanobiocomposite revealing the formation of nanobiocomposite due to the electrostatic interaction between $\mathrm{CH}$ and CNT. The IR band at $1080 \mathrm{~cm}^{-1}$ in the spectra of $\mathrm{CH}$ shifts toward the higher wavenumber $1090 \mathrm{~cm}^{-1}$ in the spectrum of both nanobiocomposites and reveals stretching vibration mode of hydroxyl groups in $\mathrm{CH}$ indicating interaction of CNT via hydrogen bonding. Moreover, the IR band at $1030 \mathrm{~cm}^{-1}$ in $\mathrm{CH}$ changes into a doublet at $1045 \mathrm{~cm}^{-1}$ and $1016 \mathrm{~cm}^{-1}$ in the spectrum of nanobiocomposites revealing that C-O-C group in glucose ring interact with CNT via weak interactions confirming the formation of $\mathrm{CH}-\mathrm{SWCNT}$ and $\mathrm{CH}-\mathrm{MWCNT}$ nanobiocomposites.

The FTIR spectra of r-IgGs and BSA immobilized CH-SWCNT/ITO and CH-MWCNT/ITO nanobiocomposite electrodes are shown in Fig. 1C and D. The presence of IR band at $1555 \mathrm{~cm}^{-1}$ observed in the spectra of $\mathrm{r}$ IgGs/CH-SWCNT/ITO (curve b, Fig. 1C) and r-IgGs/ CH-MWCNT/ITO immunoelectrode (curve b, Fig. 1D) corresponds to amide II band of rIgGs ( $\beta$-sheet, main secondary structure element of $\operatorname{IgG}$ ), indicating the presence of IgG immobilized onto the nanobiocomposite electrode. However, the presence on IR band at $1660 \mathrm{~cm}^{-1}$ and a sharp band $1572 \mathrm{~cm}^{-1}$ observed in the spectra of BSA/r-IgGs/CH-SWCNT/ITO (curve c, Fig. 1C) and BSA/r-IgGs/CH-MWCNT/ITO immunoelectrodes (curve c, Fig. 1D) corresponds to the amide II bands of BSA revealing the immobilization of BSA onto the r-IgGs/ nanobiocomposite electrode [42-43]. The new bands appearing at $\sim 1053, \sim 955$ and $\sim 850 \mathrm{~cm}^{-1}$ in the spectra of r-IgGs and BSA and immobilized CH-SW/MWCNT/ITO electrodes are attributed to the $\mathrm{N}-\mathrm{H}$ stretching mode in the amide group of proteins revealing the co-immobilization of r-IgGs and BSA onto $\mathrm{CH}-$ SWCNT/ITO and CH-MWCNT/ITO electrode.

The proposed mechanism of the fabrication of $\mathrm{CH}-\mathrm{SWCNT} / \mathrm{ITO}$ and CH-SWCNT/ITO nanobiocomposites, immobilization of r-IgGs and BSA onto both nanobiocomposites along with the biochemical reaction is shown in Fig. 1E. In our scheme, functionalized surface charged CNT interact with the cationic amine rich $\mathrm{CH}$ matrix via electrostatic interactions and hydrogen bonding (correlated by FTIR studies). To obtain an immunosensor of better sensing parameters, it is known that $\mathrm{r}$ IgG molecules should be adsorbed in a favourable orientation wherein, the $F_{c}$ (carboxyl terminated group) terminal of r-IgGs binds with nanobiocomposite electrode and its $\mathrm{F}_{\mathrm{ab}}$ (amino terminated site) terminal should be free to bind with desired antigen. Both $\mathrm{CH}-\mathrm{SWCNT} / \mathrm{ITO}$ and CH-MWCNT/ITO nanobiocomposite electrodes bind with the carboxyl groups of $\mathrm{r}-\mathrm{IgG}$ via electrostatic interactions and free amino terminal sites of r-IgG preferably bind with the carboxylic group of OTA molecules.

\section{Electrochemical studies}

Differential pulse voltammetry (DPV) studies of $\mathrm{CH} / \mathrm{ITO}$ electrode (curve a), CH-MWCNT/ITO nanobiocomposite electrode (curve b) and $\mathrm{CH}-\mathrm{MWCNT} / \mathrm{ITO}$ nanobiocomposite electrode (curve c) are shown in Fig. 2A. It is clear that the $\mathrm{CH} / \mathrm{ITO}$ electrode shows the well-defined reductive process due to the adsorption of redox [Fe(III)/ $\mathrm{Fe}(\mathrm{IV})]$ probe onto the cationic $\mathrm{CH} / \mathrm{ITO}$ electrode. The magnitude of the current response increases after the incorporation of CNT in $\mathrm{CH}$ matrix [CH-WMCNT (curve b) and CH-SWCNT nanobiocomposite (curve c)] revealing that the catalytic properties of CNTs increase the electractive surface area of $\mathrm{CH}$ resulting in improved electronic and ionic transport capacity of $\mathrm{CH}-\mathrm{CNT}$ nanobiocomposite because CNT provides a three-dimensional electron conductive network extended throughout the ion-conductive matrix of $\mathrm{CH}$. However, the magnitude of the electrochemical current response for $\mathrm{CH}-\mathrm{SWCNT/ITO}$ nanobiocomposite electrode is higher than that of the CH-MWCNT/ITO nanobiocomposite electrode due to the high local density of electronic states in SWCNT, which has been attributed to their helicity and possible topological defects resulting in enhanced electron transfer kinetics in mediator at CH-SWCNT/ITO nanobiocomposite.

These results are further confirmed using electrochemical impedance spectroscopy (EIS, Fig. 2B). EIS is a powerful technique for studying the electrochemical properties of materials at the interface of the electronically conducting electrodes. The Nyquist plot of EIS spectra includes a semicircle portion at higher frequencies corresponding to the electron transfer limited process and a linear portion at lower frequencies corresponding to the diffusion process. The electron transfer resistance $\left(\mathrm{R}_{\mathrm{CT}}\right)$ at the electrode surface is equal to the semicircle diameter, which can be used to describe the interface properties of the electrode.
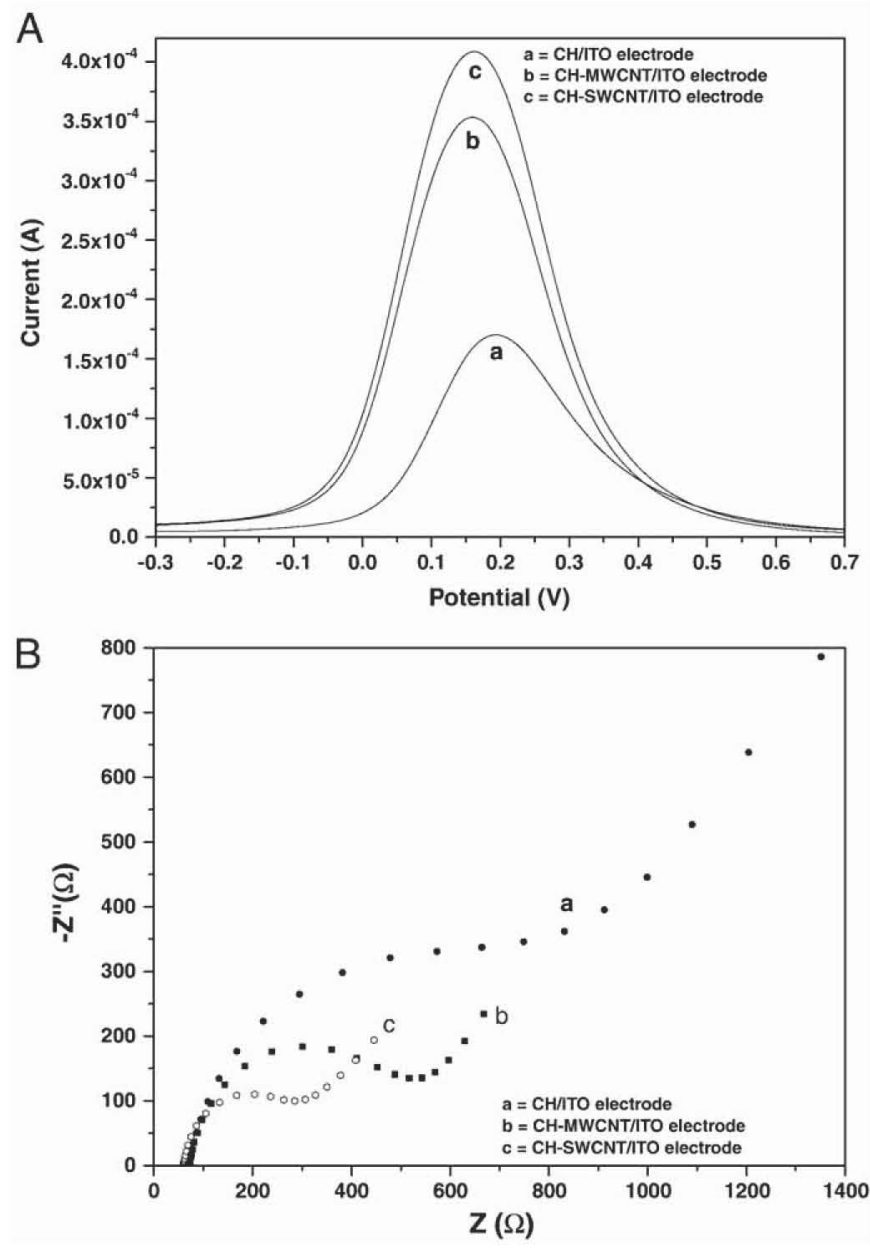

Fig. 2. A) DPV studies of $\mathrm{CH} / \mathrm{ITO}$ (curve a), $\mathrm{CH}-\mathrm{MWCNT} / \mathrm{ITO}$ nanobiocomposite (curve b) and CH-SWCNT/ITO nanobiocomposite electrodes (curve c). B) EIS studies of CH/ITO (curve a), CH-MWCNT/ITO nanobiocomposite (curve b) and CH-SWCNT/ITO nanobiocomposite electrodes (curve c). 
$\mathrm{CH} / \mathrm{ITO}$ electrode (curve a), the semicircle located near the origin is probed by higher frequencies, which means that dynamics of the electron transfer in higher frequency range is observed and the current due to voltage excitation is under kinetic control. The low frequency region, where the slope of $Z_{\text {real }}$ vs $Z_{\text {im }}$ is dominated by mass transfer of the redox species and from the interfacial region. However, diameter of the semicircle for both the CH-MWCNT/ITO (curve b) and CH-SWCNT/ ITO (curve c) nanobiocomposite electrodes decreases in comparison to that of the $\mathrm{CH} / \mathrm{ITO}$ electrode revealing the incorporation of electrical conducting $\mathrm{CNT}$ embedded in the $\mathrm{CH}$ matrix increases the electro-active surface area of $\mathrm{CH}$ resulting in enhanced electron transport. The value of the charge transfer resistance $\left(R_{C T}\right)$ for SWCNT/CH/ITO electrode is lower than that of the MWCNT/CH/ITO and this may be attributed to the open frame network and high electro catalytic properties of SWCNT that help in the easier electron transport. Hence, it is clear that the $\mathrm{CH}-$ SWCNT/ITO nanobiocomposite electrode exhibits good electrochemical behaviour than that of both the CH-MWCNT/ITO nanobiocomposite and $\mathrm{CH} / \mathrm{ITO}$ electrode and may be suitable for electrochemical biosensor due to its perfect smaller electron transfer resistance.

Fig. 3B shows the DPV studies of stepwise formation of BSA/rIgGs/CH-SWCNT/ITO (Fig. 3A) and BSA/r-IgGs/CH-MWCNT/ITO nanobiocomposite immunoelectrodes. The magnitude of the electrochemical response current further increases after the immobilization of $\mathrm{r}$-IgGs onto the $\mathrm{CH}-\mathrm{CNT} / \mathrm{ITO}$ nanobiocomposite (curve c,

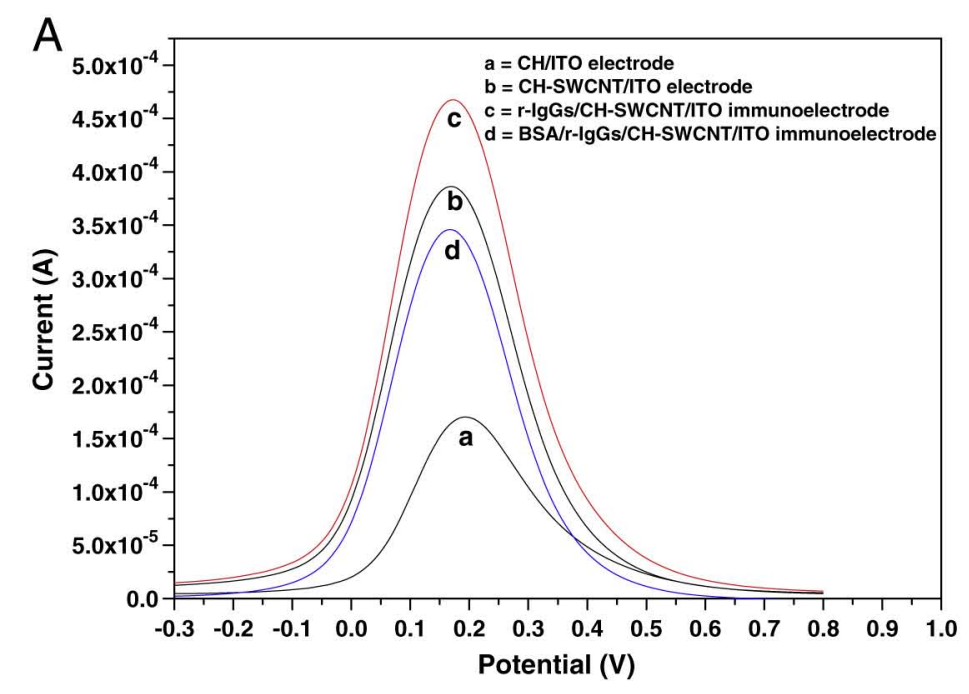

\section{$\mathrm{E}$}

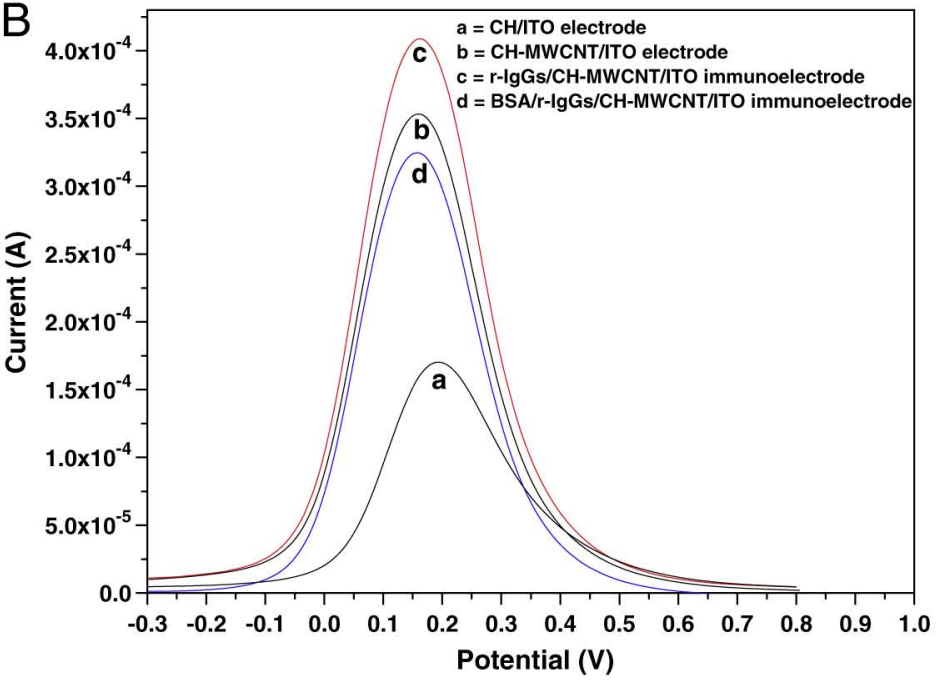

Fig. 3. A) DPV studies of $\mathrm{CH} / \mathrm{ITO}$ (curve a), $\mathrm{CH}-\mathrm{SWCNT} / \mathrm{ITO}$ nanobiocomposite (curve b), rIgGs/CH-SWCNT/ITO nanobiocomposite immunoelectrode (curve c) and BSA/r-IgGs/CHSWCNT/ITO nanobiocomposite immunoelectrode (curve d). B) DPV studies CH/ITO (curve a), CH-MWCNT/ITO nanobiocomposite (curve b), r-IgGs/CH-MWCNT/ITO nanobiocomposite immunoelectrode (curve c) and BSA/r-IgGs/CH-MWCNT/ITO nanobiocomposite immunoelectrode (curve d).
Fig. 3A \& B) revealing that $\mathrm{CH}-\mathrm{CNT}$ provides a good environment for IgGs immobilization and available non-binding sites on r-IgGs making r-IgGs/CH-CNT/ITO immunoelectrode positively charged resulting in both increased diffusion of electrons and electron transport between r-IgGs and electrode. However, after the immobilization of BSA onto the r-IgGs/CH-CNT/ITO immunoelectrode (curve d, Fig. 3A \& B) the magnitude of the current decreases due to blocking of the non-binding sites of r-IgGs/CH-CNT/ITO immunoelectrode resulting in hindered electron transfer between the medium and electrode confirming the immobilization of BSA onto r-IgGs/CHCNT/ITO immunoelectrode. The similar results have been obtained in the $\mathrm{CV}$ studies (data not shown) of $\mathrm{CH} / \mathrm{ITO}$ electrode, $\mathrm{CH}-\mathrm{SW} / \mathrm{MW}$ CNT/ITO nanobiocomposite electrode, $\mathrm{r}-\mathrm{IgGs} / \mathrm{CH}-\mathrm{SW} / \mathrm{MW}-\mathrm{CNT} / \mathrm{ITO}$ immunoelectrode and BSA/r-IgGs/CH-SW/MWCNT/ITO immunoelectrode). Interestingly, the magnitude of the current response of $\mathrm{BSA} / \mathrm{r}-\mathrm{IgGs} / \mathrm{CH}-\mathrm{SWCNT} / \mathrm{ITO}$ immunoelectrode is higher than that of the BSA/r-IgGs/CH-MWCNT/ITO immunoelectrode, due to the threedimensional arrangement and electro catalytic properties of SWCNT in the $\mathrm{CH}$ matrix to increase the electro-active surface area of $\mathrm{CH}-$ SWCNT nanobiocomposite for high loading of r-IgGs resulting in improved electrochemical properties of BSA/r-IgGs/CH-SWCNT/ITO immunoelectrode.

The surface concentrations of ionic redox species on the $\mathrm{CH} /$ ITO electrode and CH-SWCNT/ITO nanobiocomposite electrodes, CH-MWCNT/ITO nanobiocomposite electrode have been estimated using Eq. (1) [40].

$i_{p}=0.227 n F A C_{0}^{*} k^{0} \exp \left[\frac{-\alpha n_{a} F}{R T}\left(E_{p}-E_{0}^{\prime}\right)\right]$

where, $i_{p}$ is the anodic peak current, $n$ is the number of electrons transferred (1), $F$ is the Faraday constant $\left(96485.34 \mathrm{Cmol}^{-1}\right), A$ is surface area $\left(0.25 \mathrm{~cm}^{-2}\right), R$ is the gas constant $\left(8.314 \mathrm{~J} \mathrm{~mol}^{-1} \mathrm{~K}^{-1}\right)$, $C_{0}^{*}$ is the surface concentration of the ionic species of film surface $\left(\mathrm{mol} \mathrm{cm}{ }^{-3}\right), E_{p}$ is the peak potential and $E_{0}^{\prime}$ is the formal potential. $-\alpha n_{a} F / R T$ and $k^{0}$ (rate constant) correspond to the slope and intercept of $\ln \left(i_{p}\right)$ vs the $E_{p}-E_{0}^{\prime}$ curve at different scan rates. It may be noted that surface concentration of CH-SWCNT/ITO nanobiocomposite electrode $\left(3.2 \times 10^{-6} \mathrm{~mol} \mathrm{~cm}^{-3}\right)$, and $\mathrm{CH}-\mathrm{MWCNT} / \mathrm{ITO}$ nanobiocomposite electrode $\left(2.4 \times 10^{-6} \mathrm{~mol} \mathrm{~cm}^{-3}\right)$ are higher than that of the $\mathrm{CH} / \mathrm{ITO}\left(1.4 \times 10^{-6} \mathrm{~mol} \mathrm{~cm}^{-3}\right)$. The increased surface concentration of redox species onto the $\mathrm{CH}-\mathrm{CNT} / \mathrm{ITO}$ nanobiocomposite electrode reveals increased redox moieties that are available for oxidation leading to a higher faradic current wherein, the presence of CNT results in increased electron transport between redox species and electrode.

Fig. 4A and B show result of CV studies carried out on both BSA/rIgGs/ CH-SWCNT/ITO (Fig. 3A) and BSA/r-IgGs/CH-MWCNT/ITO (3B) nanobiocomposite immunoelectrodes as a function of scan rate (10$100 \mathrm{mV} / \mathrm{s}$ ). It can be seen that potential and magnitude of the response currents are dependent on the scan rate that exhibits a linear relationship with the sweep rate (inset: Fig. $3 \mathrm{~A}$ and $\mathrm{B}$ ) revealing that the electrochemical reaction is a diffusion-controlled process and facile electron transfer between medium and electrode [35].

The effect of $\mathrm{pH}$ on both BSA/r-IgGs/CH-SWCNT/ITO and BSA/r-IgGs/ CH-MWCNT/ITO immunoelectrodes has been carried out using DPV technique (data not shown). It is observed that the magnitude of the current increases in the $\mathrm{pH}$ range from 6.0 to 7.0. The magnitude of the current has been found to decrease by further increasing the $\mathrm{pH}$ from 7.0 to 8.0. This suggests that both electrode show maximum activity at $\mathrm{pH} 7$ at which r-IgGs and BSA retain their natural structure and do not denature. Thus all the experiments have been conducted at $\mathrm{pH}$ 7.0. The DPV analysis of $\mathrm{CH} / \mathrm{ITO}$ electrode, $\mathrm{CH}-\mathrm{CNT} / \mathrm{ITO}$ nanobiocomposite electrode and BSA/r-IgGs/CH-SW/MW CNT/ITO immunoelectrodes have been repeated several times ( 20 times) on the same film surface 

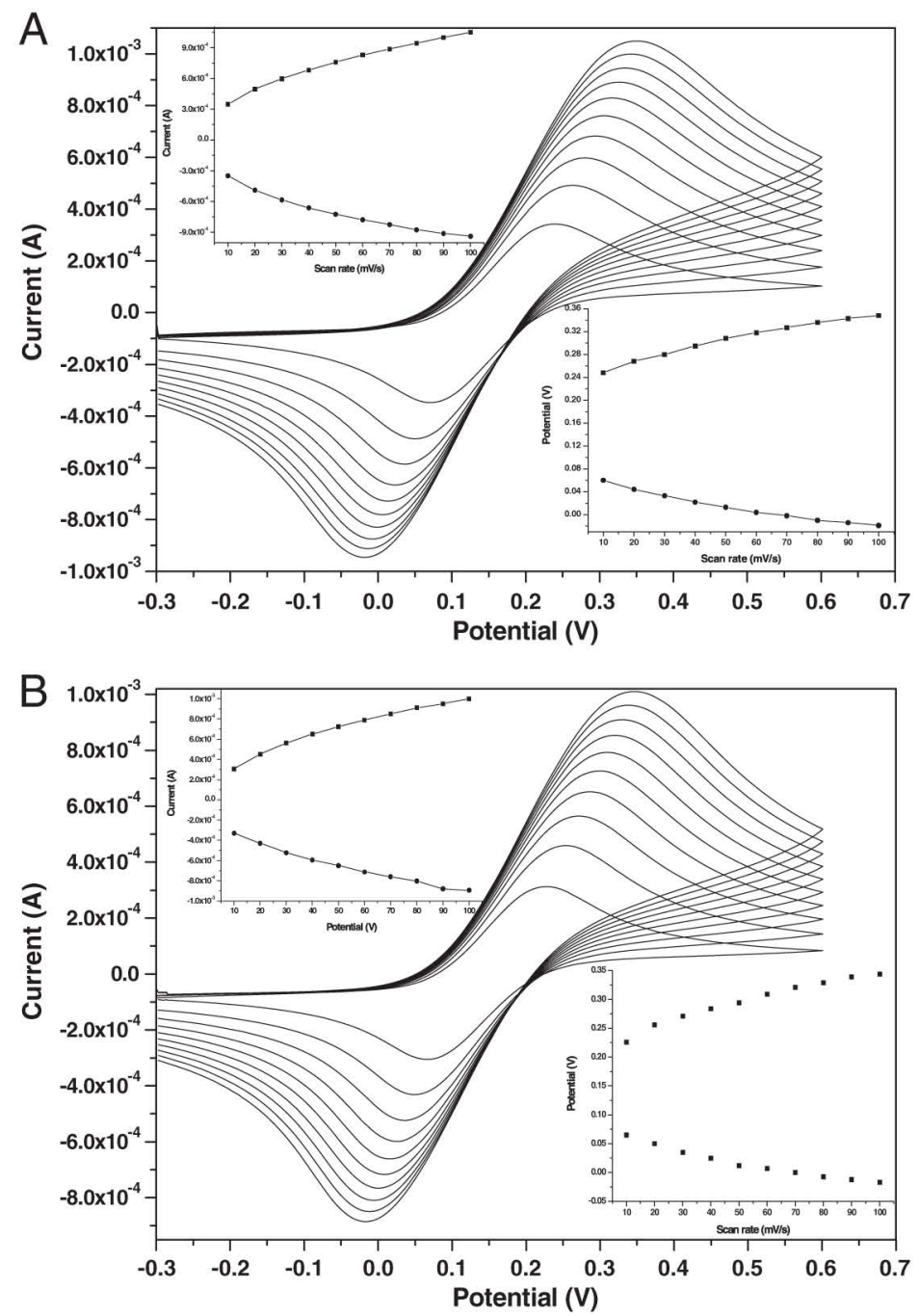

Fig. 4. A) CV studies of BSA/r-IgGs/CH-SWCNT/ITO nanobiocomposite immunoelectrode as function of scan rate $(10-100 \mathrm{mV} / \mathrm{s})$. B) CV studies of BSA/r-IgGs/CH-MWCNT/ITO nanobiocomposite immunoelectrode as function of scan rate $(10-100 \mathrm{mV} / \mathrm{s})$.

in electrolyte and different films have been prepared from the same stock solution and the results are reproducible (with $4 \%$ error).

\section{Electrochemical response studies}

The electrochemical response of both the BSA/r-IgGs/CH-MWCNT/ ITO (Fig. 5A) and BSA/r-IgGs/CH-SWCNT/ITO (Fig. 5A) immunoelectrodes has been investigated as a function of OTA concentration using DPV technique in PBS solution $\{50 \mathrm{mM}$ PBS ( $\mathrm{pH} 7,0.9 \% \mathrm{NaCl}$ ) containing $5 \mathrm{mM}\left[\mathrm{Fe}(\mathrm{CN})_{6}\right]^{3-/ 4-}$. The magnitude of the electrochemical current response is found to be increase on the addition of OTA $(0.25-6 \mathrm{ng} / \mathrm{dL})$.

This can be attributed to the formation of antigen-antibody complex between OTA and r-IgGs on electrode surface that acts as electron transfer-accelerating layer resulting in the enhanced electron transfer between r-IgGs and electrode via $\mathrm{CH}-\mathrm{CNT}$ nanobiocomposite. Inset in Fig. 5A shows the calibration curve of BSA/r-IgGs/ $\mathrm{CH}-\mathrm{MWCNT/ITO}$ immunoelectrode obtained as a function of OTA concentration. A linear relationship between the magnitude of current and OTA concentration can be fitted to the experimental points from 0.5 to $6 \mathrm{ng} \mathrm{dL}^{-1}$ and follows Eq. (2).

$I(A)=3.18 \times 10^{-4}(A)+15 \mu \mathrm{A} d L n g^{-1} \times\left[\right.$ OTA concentration $\left.\left(n g d L^{-1}\right)\right]$

BSA/r-IgGs/CH-MWCNT/ITO immunosensor exhibits sensing characteristics such as low detection limit $\left(0.25 \mathrm{ng} \mathrm{dL}^{-1}\right)$, reproducibility
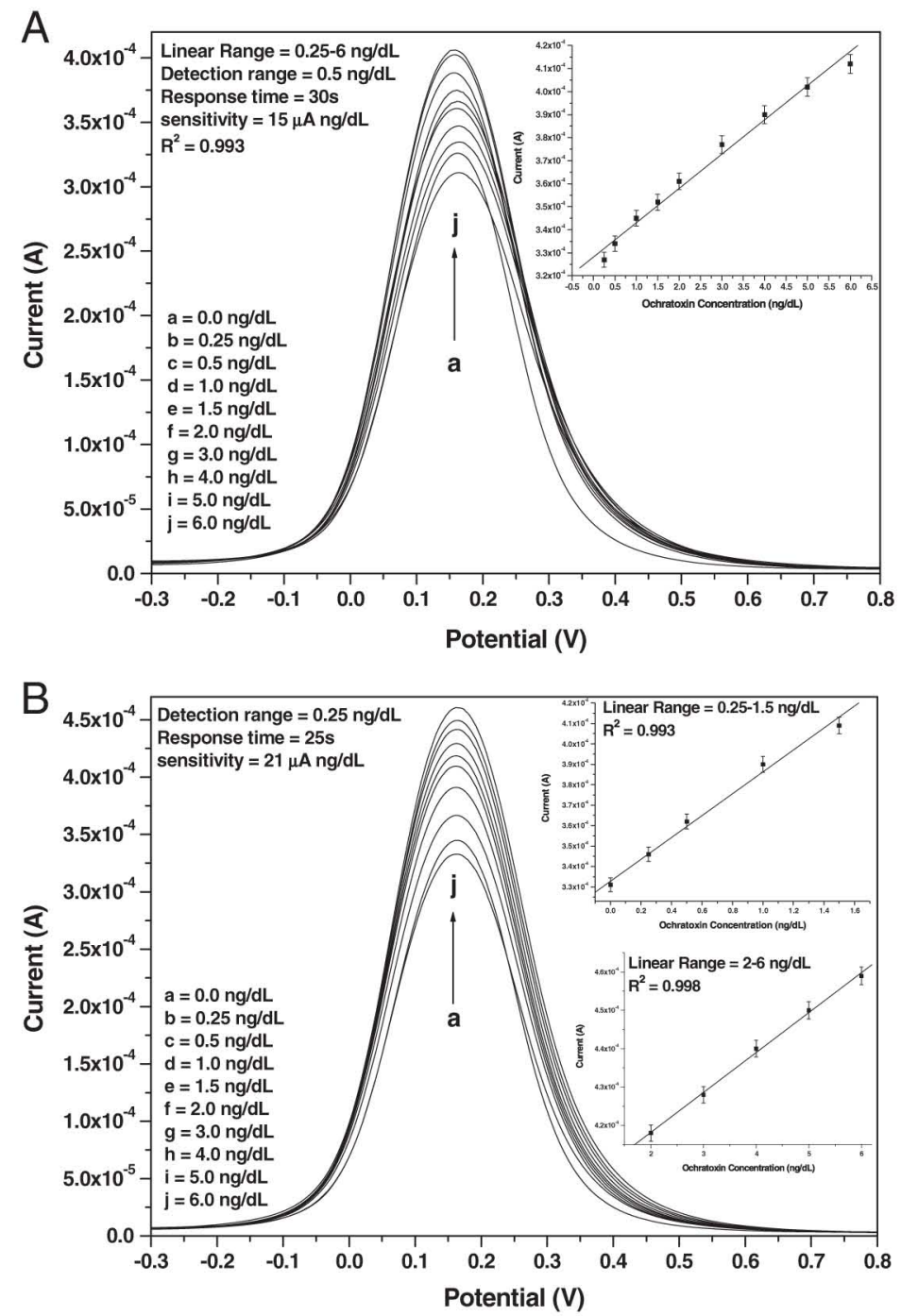

Fig. 5. Electrochemical response studies both BSA/r-IgGs/CH-MWCNT/ITO (A) and BSA/ $\mathrm{r}$-IgGs/CH-SWCNT/ITO nanobiocomposite immunoelectrodes (B) as function of OTA concentration $(0.25-6 \mathrm{ng} / \mathrm{dL})$.

(15 times), long term stability ( 80 days) and sensitivity ( $15 \mu \mathrm{A} /$

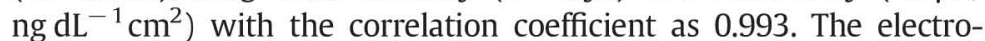
chemical response of BSA/r-IgGs/CH-SWCNT/ITO immunosensor (Fig. 5B) is also measured as a function of OTA concentration (0.25$6 \mathrm{ng} / \mathrm{dL}^{-1}$ ) with improved sensitivity calculated from the slope of curve has been found to be $21 \mu \mathrm{A} / \mathrm{ng} \mathrm{dL}^{-1} \mathrm{~cm}^{2}$. It has been shown that good linearity is found in the low concentration range $\left(0.25-1.5 \mathrm{ng} \mathrm{dL}^{-1}\right)$ and the current follows Eq. (3) with correlation coefficient of 0.995 .

$\mathrm{I}(A)=3.3 \times 10^{-4}(A)+53.5 \times \mu \mathrm{AdLng}^{-1}$

$$
\times\left[\text { OTA concentration }\left(\mathrm{ng} \mathrm{dL}^{-1}\right)\right] \text { with } R^{2}=0.995
$$

As OTA concentration increases from 2 to $6 \mathrm{ng} \mathrm{dL}^{-1}$, the observed response current starts to level off due to the restriction of the r-IgGs-OTA reaction that changes the order of reaction. Interestingly, in the OTA concentration range $\left(2-6 \mathrm{ng} \mathrm{dL}^{-1}\right)$ the change in the electrochemical response current follows Eq. (4).

$$
\begin{aligned}
\mathrm{I}(A)= & 3.97 \times 10^{-4}(A)+10.5 \times \mu A \mathrm{dLng}^{-1} \\
& \times\left[\text { OTA concentration }\left(\mathrm{ng} \mathrm{dL}^{-1}\right)\right] \text { with } \mathrm{R}^{2}=0.998
\end{aligned}
$$

However, due to the open frame network morphology and high electrocatalytic properties of SWCNT than that of the MWCNT, BSA/rIgGs/CH-SWCNT/ITO immunosensor shows improved sensing 
characteristics. The reproducibility of response of the immunoelectrode has been investigated at $1 \mathrm{ng} \mathrm{dL}-1$ OTA concentration with the time interval of 7 days. No significant decrease in the current is observed revealing that immunosensor can be used $\sim 15$ times with a response time of $25 \mathrm{~s}$ and a shelf life $\sim 90$ days (data not shown). The apparent value of the association constant $(\mathrm{Ka})$ obtained as $4 \times 10^{12} \mathrm{Lmol}^{-1}$ using a Lineweaver-Burke-like plot indicates high affinity of $\mathrm{r}-\mathrm{IgGs}$ towards OTA due to prevalent electrostatic interactions. The high affinity of the BSA/r-IgGs/CH-SWCNT/ITO immunoelectrode can be attributed to favourable conformation of r-IgGs and increased loading of $\mathrm{r}$-IgGs provided by the microenvironment of the $\mathrm{CH}$ and functionalized CNT nanobiocomposite platform [41].

\section{Conclusions}

In summary, r-IgGs and BSA have been co-immobilized onto $\mathrm{CH}-\mathrm{SWCNT} / \mathrm{ITO}$ and $\mathrm{CH}-\mathrm{MWCNT} / \mathrm{ITO}$ nanobiocomposite electrodes for OTA detection. Due to the open frame network and high electrochemical properties of SWCNT, BSA/r-IgGs/CH-SWCNT/ITO immunoelectrode exhibits better improvement than that of the CH-MWCNT nanobiocomposite immunoelectrode. BSA/r-IgGs/ $\mathrm{CH}-\mathrm{SWCNT} / \mathrm{ITO}$ immunosensor may find potential application for the detection of other clinically important antigens such as aflatoxin, ochratoxin B, citrinin, ergot alkaloids, fumonisins, patulin, trichothecenes, and zearalenone etc.

\section{Acknowledgements}

We thank to Porf. R. C. Budhani, Director, National Physical Laboratory, New Delhi, India for providing facilities. A K and PRS are thankful to Department of Science and Technology (DST), India for the award of Senior Research Associate ship and Senior Research Fellowship.

\section{References}

[1] S. Iijima, Nature 354 (1991) 56.

[2] M. Ajayan, Chem. Rev. 99 (1999) 1787.

[3] J. Wang, Electroanalysis 17 (2007) 7.

[4] M.D. Rubianes, G.A., Electroanalysis 17 (2005) 73.
[5] M. Trojanowicz, Trends Anal. Chem. 25 (2006) 480.

[6] A. Merkoci, M. Pumera, X. Llopis, B. Perez, M.D. Valle, S. Alegret, Trends Anal. Chem. 24 (2005) 826.

[7] S. Sotiropoulou, N.A. Chaniotakis, Anal. Bioanal. Chem. 375 (2003) 103.

[8] S. Roy, H. Vedala, W. Choi, Nanotechnology 17 (2006) S14.

[9] Y. Yao, K.K. Shiu, Anal. Bioanal. Chem. 387 (2007) 303.

[10] M. Gao, L. Dai, G.G. Wallace, Electroanalysis 15 (2003) 1089.

[11] M. O'Connor, S.N. Kim, A.J. Killard, R.J. Forster, M.R. Smyth, F. Papadimitrakopoulosb, J.F. Rusling, Analyst 129 (2004) 1176.

[12] C. Dhand, S.K. Arya, M. Datta, B.D. Malhotra, Anal. Biochem. 383 (2008) 194.

[13] C. Dhand, S.K. Arya, S.P. Singh, B.P. Singh, M. Datta, B.D. Malhotraa, Carbon 46 (2008) 1727.

[14] P.J. Britto, K.S.V. Santhanam, P.M. Ajayan, Bioelectrochem. Bioenerg. 41 (1996) 121.

[15] M. Musameh, J. Wang, A. Merkoci, Y. Lin, Electrochem. Commun. 4 (2002) 743.

[16] J. Wang, M. Li, Z. Shi, N. Li, Z. Gu, Electroanalysis 14 (2002) 225.

[17] J. Wang, M. Li, Z. Shi, N. Li, Z. Gu, Anal. Chem. 74 (2002) 1993.

[18] Z. Wang, J. Liu, Q. Liang, Y. Wang, G. Luo, Analyst 127 (2002) 653.

[19] J. Wng, M. Li, Z. Shi, N. Li, Z. Gu, Electrochimica Acta 47 (2001) 651.

[20] R. Antiochia, I. Lvagnini, P. Pastore, F. Magno, Bioelectrochemistry 64 (2004) 157.

[21] N.S. Lawrence, R.D. Deo, J. Wang, Talanta 63 (2004) 443.

[22] J. Wang, A.N. Kawdw, M. Musameh, Analyst 129 (2003) 125.

[23] G.M. Spinks, S.R. Shin, G.G. Wallace, P.G. Whitten, S.I. Kim, S.J. Kim, Sensors Actuators B 115 (2006) 678.

[24] Y. Liu, J. Tang, X. Chen, J.H. Xin, Carbon 43 (2005) 3178.

[25] X.L. Luo, J.J. Xu, J.L. Wang, H.Y. Chen, Chem. Commun. (2005) 2169.

[26] M. Zhang, W. Gorski, J. Am. Chem. Soc. 127 (2005) 2058.

[27] X. Wei, J. Cruz, W. Gorski, Anal. Chem. 74 (2002) 5039.

[28] M. Zhang, A. Smith, W. Gorski, Anal. Chem. 76 (2004) 5045.

[29] J. Li, Qian Liu, Yingju Liu, Shanchao Liu, Shouzhuo Yao, Anal. Biochem 346 (2005) 107.

[30] Y. Liu, M. Wang, Feng Zhao, Zhiai Xu, Shaojun Dong, Biosens. Bioelectron 21 (2005) 984.

[31] X. Cui, C.M. Li, J. Zang, S. Yu, Biosens. Bioelectron. 22 (2007) 3288.

[32] Y. Wang, W. Wei, J. Zeng, X. Liu, X. Zeng, Microchim. Acta 160 (2008) 253.

[33] P.R. Solanki, A. Kaushik, A.A. Ansari, A. Tiwari, B.D. Malhotra, Sens. Actuators B 137 (2009) 727.

[34] J. Lin, C. He, L. Zhang, S. Zhang, Anal. Biochem. 384 (2009) 30.

[35] A. Kaushik, P.R. Solanki, A.A. Ansaril, S. Ahmad, B.D. Malhotra, Nanotechnology 20 (2009) 055105.

[36] A. Kaushik, P.R. Solanki, A.A. Ansari, S. Ahmad, B.D. Malhotra, Electrochem. Commun. 10 (2008) 1364.

[37] R.B. Mathur, S. Seth, C. Lal, R. Rao, B.P. Singh, T.L. Dhami, A.M. Rao, Carbon 45 (2007) 132.

[38] R.B. Mathur, S. Chatterjee, B.P. Singh, Compos. Sci. Technol. 68 (2008) 1608.

[39] K. Kan, T. Xia, L. Li, H. Bi, H. Fu, K. Shi, Nanotechnology 20 (2009) 185502.

[40] A. Kaushik, R. Khan, P. Pandey, J. Alam, S. Ahmad, B.D. Malhotra, Biosens. Bioelectron. 24 (2008) 676.

[41] J. Kuby, in: Richard A. Goldsby, Thomas J. Kindt, Barbara A. Osborne (Eds.), Kuby Immunology 4e, 4th ed., W. H. Freeman \& Company, January 152000. 